\title{
Trastuzumab with FLOT Regimen for the Perioperative Treatment of Resectable HER2 + Advanced Gastric Cancer: A Retrospective Study
}

This article was published in the following Dove Press journal: Cancer Management and Research

\author{
Gangling Tong ${ }^{1 * *}$ \\ Shuluan $\mathrm{Li}^{2, *}$ \\ Lin $\operatorname{Lin}^{3, *}$ \\ Lirui $\mathrm{He}^{\prime}$ \\ Li Wang' \\ Guoqing Lv' \\ Ruinian Zheng ${ }^{4}$ \\ Shubin Wang' \\ 'Department of Oncology, Peking \\ University Shenzhen Hospital, Shenzhen, \\ Guangdong 518036, People's Republic of \\ China; ${ }^{2}$ National Cancer Center/National \\ Clinical Research Center for Cancer/ \\ Cancer Hospital, Chinese Academy of \\ Medical Sciences and Peking Union Medical \\ College, Shenzhen, Guangdong 518116, \\ People's Republic of China; ${ }^{3}$ Department of \\ Oncology, Shenzhen Second \\ Peoples Hospital, Shenzhen, Guangdong \\ 518036, People's Republic of China; \\ ${ }^{4}$ Department of Oncology, Affiliated \\ Dongguan People's Hospital, Southern \\ Medical University, Dongguan, Guangdong \\ 523000, People's Republic of China \\ *These authors contributed equally to this \\ work
}

Correspondence: Ruinian Zheng Department of Oncology, Affiliated Dongguan People's Hospital, Southern Medical University, Wandao Road

(South), Xinguyong Village, Wanjiang

District, Dongguan, Guangdong 523000,

People's Republic of China

Tel +86-769-2863707I

Email 13450023449@I63.com

Shubin Wang

Department of Oncology, Peking University Shenzhen Hospital, Lianhua

Road (North), Futian District, Shenzhen, Guangdong 518036, People's Republic of China

$\mathrm{Tel} / \mathrm{Fax}+86-755-83923333$

Email wangshubin2013@I63.com
Background: The aim of this study was to evaluate the efficacy and safety of trastuzumab, combined with the FLOT regimen, in the perioperative treatment of resectable HER-2-positive advanced gastric cancer.

Methods: Overall, 45 patients were divided into two groups; 29 patients in the experimental group were treated with trastuzumab combined with FLOT and 16 patients in the control group were treated with FLOT alone. The primary endpoint was objective response rate (ORR), and the secondary endpoints were disease control rate (DCR), tumor regression grade (TRG), surgical margin, side effects, and overall survival.

Results: In the experimental and control groups, ORR was $72.4 \%$ and $43.8 \%(p=0.226)$, DCR was $89.7 \%$ and $87.5 \%$, R0 resection rate was $96.5 \%$ and $93.7 \%$, total/subtotal tumor regression grade was $17.2 \%$ and $6.3 \%$, partial tumor regression grade was $27.6 \%$ and $18.7 \%$ ( $p=0.468$ ), and 2-year survival rate was $78.1 \%$ and $73.9 \%(p=0.932)$, respectively. The common side effects were agranulocytosis and vomiting. There was no significant difference between the two groups.

Conclusion: Trastuzumab combined with FLOT has a good curative effect and safety profile in the perioperative treatment of patients with resectable HER-2-positive advanced gastric cancer. In addition, trastuzumab + FLOT had the same result as FLOT alone, as there was no significant benefit with the addition of $\mathrm{T}$ in the group studied.

Keywords: gastric cancer, HER2, perioperative chemotherapy, trastuzumab

\section{Introduction}

Gastric cancer is one of the most common malignant tumors worldwide and ranks third in terms of mortality rate. In recent years, with the diversification of treatment methods, patients with pathological stage I disease have a cure rate of $90 \%$; however, the prognosis of advanced gastric cancer remains poor. Advanced gastric cancer accounts for about $80 \%$ of gastric cancer patients in China. Surgery is an important treatment modality for achieving cure among patients with advanced gastric cancer. However, there is still a high risk of metastasis and recurrence, and the long-term survival rate is still low. ${ }^{1}$ According to the clinical study of MAGIC, ECF can improve the overall survival (OS) of patients with operable gastric cancer. The 5-year survival rate of patients who underwent an operation only was $23 \%$, while that of patients with combined treatment was $36 \%(p=0.0009) .^{2}$ The FLOT4-AIO clinical study found that FLOT was superior to ECF/ECX during the perioperative period in patients with locally advanced gastric or gastroesophageal junction adenocarcinoma. The OS was 50 
months and 35 months (HR, $0.77 ; P=0.012){ }^{3}$, respectively. Therefore, neoadjuvant therapy can be the first choice for advanced gastric cancer. Previous studies have reported that HER-2 protein expression level in those with gastric cancer was between $7.3 \%$ and $22.1 \%$. ${ }^{4}$ HER-2 expression in gastric cancer was more invasive and the prognosis of patients was worse, but HER-2 was also an effective predictor of trastuzumab treatment. ${ }^{5}$ A stage III ToGA clinical study showed that the efficacy of trastuzumab combined with an XP/FP regimen in patients with HER-2-positive advanced esophagogastric junction or gastric cancer was significantly better than that of patients receiving only chemotherapy. Median OS was 13.8 months (95\% confidence interval [CI] 12-16) for patients assigned to the trastuzumab plus chemotherapy arm compared with 11.1 months (95\% CI 10-13) in the chemotherapy group (hazard ratio [HR] 0.74; 95\% CI 0.60-$0.91 ; p=0.0046)$. Especially in the patients with HER-2 immunohistochemistry $2+$ and FISH positive or the patients with HER-2 immunohistochemistry $3+$, the survival improvement was more obvious, with the median OS better in the experimental arm (16.8 vs 11.8 months, HR: 0.65$){ }^{6}$ Japan's JACOB clinical study found that trastuzumab combined with an XP/PF regimen in the treatment of those with HER-2-positive metastatic gastric cancer demonstrated an effective rate as high as $48.3 \%$. In addition, it was found that the combination of pertuzumab did not improve the prognosis of these patients, as the median OS was $17 \cdot 5$ months [95\% CI 16.2-19.3] in the pertuzumab group and $14 \cdot 2$ months $[12 \cdot 9-15 \cdot 5]$ in the control group (hazard ratio $0 \cdot 84$ [95\% CI 0.71-1.00]; $\mathrm{p}=0 \cdot 057){ }^{7}$ Another study found that trastuzumab in combination with oxaliplatin//capecitabine in first-line treatment of HER2-positive advanced gastric cancer was associated with a median progression-free survival of 9.2 months (95\% confidence interval [CI]: 6.5-11.6) and a median OS of 19.5. There was also a reported favorable toxic profile and high efficacy of XELOX/ trastuzumab. ${ }^{8}$ However, data concerning the use of trastuzumab plus chemotherapy as perioperative treatment of resectable HER2 + advanced gastric cancer are still limited. There is currently a Phase II clinical trial of PETRARCA which is a multicenter, randomized, controlled, open-label study of FLOT vs FLOT/Herceptin/Pertuzumab for perioperative therapy of adenocarcinoma of the stomach and gastroesophageal junction expressing HER-2; however, the results have not been reported. Therefore, it is not clear whether trastuzumab combined with FLOT is better than FLOT alone in the perioperative treatment of advanced gastric cancer. In this study, we retrospectively analyzed and discussed the value of this treatment modality in perioperative treatment of HER2-positive gastric cancer in operable advanced stage, to enrich the basis of clinical treatment.

\section{Materials and Methods Case Selection and General Information}

From January 2016 to January 2019, the clinical data of 45 patients with operable advanced HER2-positive gastric cancer who were treated at Peking University Shenzhen Hospital, Shenzhen Hospital/Cancer Hospital/Chinese Academy of Medical Sciences, Shenzhen Second People's Hospital, and Dongguan People's Hospital were collected. All cases were confirmed via pathology. The inclusion criteria were: 1) Age 18-70 years. 2) Gastric adenocarcinoma confirmed via gastroscopic biopsy with immunohistochemistry (IHC) as HER2 $(3+)$ or IHC (2+) and fluorescence in situ hybridization positive. The HER2 staining score criteria referred to the gastric cancer HER2 guidelines. 3) Computed tomography (CT) scan and/or magnetic resonance imaging (MRI) combined endoscopic ultrasonography clearly showed advanced gastric cancer without distant metastasis, and with tumor invasion depth $\geq \mathrm{cT} 1$ and $\mathrm{cN}+, \mathrm{cM} 0$. The lesions could be measured and the TNM (American Joint Commission on Cancer) stage criteria were used. 4) After a multidisciplinary team discussion, patients could be treated surgically. 5) The Eastern Cooperative Oncology Group (ECOG) performance score was $0-2$. The survival time was $>3$ months. 6 ) The patients were newly treated with left ventricular ejection fraction $>50 \%$, no history of congestive heart failure and myocardial infarction, no obvious abnormalities in blood routine, liver, and kidney function, no serious complications, and normal function of other important organs. 7) All patients had complete clinical data and informed consent (the general characteristics are shown in Figure 1 and Table 1).

\section{Therapeutic Approach}

Forty-five patients with HER2-positive advanced gastric cancer were divided into two groups. Patients in the experimental group were treated with T + FLOT $(n=29)$ and those in the control group with FLOT $(n=16)$. The details of the FLOT regimen are as follows: docetaxel (Sanofi), $50 \mathrm{mg} / \mathrm{m}^{2}$ via intravenous drip; oxaliplatin (Sanofi), $85 \mathrm{mg} / \mathrm{m}^{2}$ via intravenous drip for $2 \mathrm{hrs}$; calcium folinate (Hengrui), $200 \mathrm{mg} / \mathrm{m}^{2}$ via intravenous drip for $2 \mathrm{hrs}$; 5-fluorouracil (Hengrui), $2600 \mathrm{mg} / \mathrm{m}^{2}$ via continuous pump for $24 \mathrm{hrs}$ 


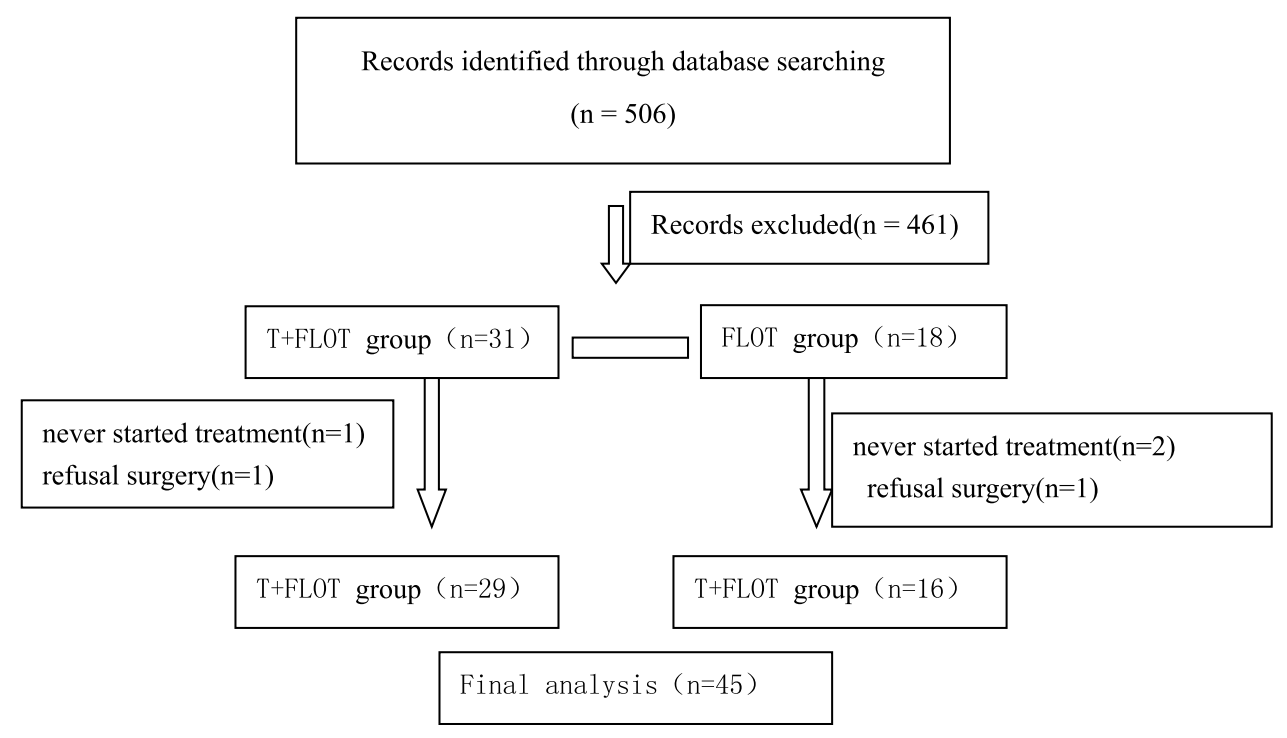

Figure I Protocol overview.

on day 1 , repeated for 2 weeks (4 cycles); trastuzumab (Roche), $8 \mathrm{mg} / \mathrm{kg}$ in the first intravenous drip, $6 \mathrm{mg} / \mathrm{kg}$ in the second on day 1, repeated for 3 weeks, 3 cycles in total. CT scan or MRI was performed every 4 weeks to evaluate the effect. Surgical treatment could be considered for tumor enlargement, and chemotherapy could be considered 3 weeks pre- or postoperatively. According to the tumor location, proximal, distal, or total gastrectomy combined with D2 dissection could be selected. Follow-up treatment options were pursued according to the pathological results. Patients with curative effect (stable disease [SD] or better) were treated with the original regimen (FLOT 8 cycles, trastuzumab 6 cycles). Patients with curative effect (progressive disease [PD]) could opt for the mFOLFOX6 regimen (at least 3 months). The dosages of chemotherapeutic drugs were adjusted according to the patients' tolerance. The adverse effects were evaluated according to the World Health Organization (WHO) criteria.

\section{Evaluation Indicators}

The primary endpoint was objective response rate (ORR), and the secondary endpoints were disease control rate (DCR), tumor regression grade (TRG), surgical margin, adverse effects, and OS. CT or MRI examinations were performed before and during neoadjuvant chemotherapy, and the clinical stages were determined. According to the stages of cTNM before neoadjuvant chemotherapy and pTNM after neoadjuvant chemotherapy, the effect of phase reduction was evaluated. According to the Response Evaluation Criteria in Solid Tumors, the therapeutic effects were divided into complete response $(\mathrm{CR})$, partial response (PR), SD, and PD. The ORR was calculated by summing the $\mathrm{CR}$ and PR, and the DCR was calculated by summing the CR, PR, and SD. According to the Becker classification criteria, TRG1 referred to complete or subtotal regression (1a: complete, no residual tumor cells; 1b: subtotal, less than $10 \%$ residual tumor cells in each tumor bed), TRG2 referred to partial regression (residual tumor cells in each tumor bed ranging from $10 \%$ to $50 \%$ ) and TRG3 referred to little or no regression (residual tumor cells $>50 \%$ in each tumor bed).

\section{Statistical Analysis}

The rates were compared using the chi-squared test. The survival rate and univariate analysis of prognosis were assessed via the Kaplan-Meier method. The statistical significance of differences in survival was determined via the Log-rank test. All statistical analyses were performed using SPSS version 22.0 (IBM Corp., Armonk, NY, USA). Analysis items with $\mathrm{P}<0.05$ were considered statistically significant.

\section{Results Objective Response Rate and Disease Control Rate}

Forty-five patients with operable HER2-positive advanced gastric cancer completed neoadjuvant treatment. The efficacy of the experimental group and the control group is shown in Table 2. Among 29 patients in the experimental 
Table I General Characteristics of 45 Patients with HER2Positive Advanced Gastric Cancer

\begin{tabular}{|c|c|c|c|}
\hline $\begin{array}{l}\text { Group Clinical } \\
\text { Characteristics }\end{array}$ & $\begin{array}{l}\text { T + FLOT, } \\
\text { n (\%) }\end{array}$ & $\begin{array}{l}\text { FLOT, } \\
\text { n (\%) }\end{array}$ & $P$ value \\
\hline $\begin{array}{l}\text { Age, median (years) } \\
\quad<60 \\
\geq 60\end{array}$ & $\begin{array}{l}59.7(32-65) \\
22(75.9) \\
7(24.1)\end{array}$ & $\begin{array}{l}49.7(33-65) \\
13(81.3) \\
3(18.7)\end{array}$ & 0.677 \\
\hline $\begin{array}{l}\text { Gender } \\
\text { Male } \\
\text { Female }\end{array}$ & $\begin{array}{l}16(55.2) \\
13(44.8)\end{array}$ & $\begin{array}{l}\text { II (68.7) } \\
5(31.3)\end{array}$ & 0.373 \\
\hline $\begin{array}{l}\text { ECOG score } \\
0 \\
1 \\
2\end{array}$ & $\begin{array}{l}\text { II (68.7) } \\
5(17.2) \\
3(10.4)\end{array}$ & $\begin{array}{l}21(72.4) \\
5(31.3) \\
0(0)\end{array}$ & 0.275 \\
\hline $\begin{array}{l}\text { Location } \\
\text { Fundus of stomach } \\
\text { Gastric body } \\
\text { Antrum of stomach } \\
\text { Whole stomach }\end{array}$ & $\begin{array}{l}3(10.4) \\
4(13.8) \\
19(65.4) \\
3(10.4)\end{array}$ & $\begin{array}{l}3(18.7) \\
2(12.5) \\
10(62.5) \\
1(6.3)\end{array}$ & 0.857 \\
\hline $\begin{array}{l}\text { Pathological } \\
\text { differentiation } \\
\text { High differentiation } \\
\text { Medium differentiation } \\
\text { Poor differentiation or } \\
\text { undifferentiation }\end{array}$ & $\begin{array}{l}7(24.2) \\
9(31.0) \\
13(44.8)\end{array}$ & $\begin{array}{l}3(18.7) \\
6(37.5) \\
7(43.8)\end{array}$ & 0.875 \\
\hline $\begin{array}{l}\text { Clinical stage } \\
\text { II } \\
\text { IIIA } \\
\text { IIIB } \\
\text { IIIC }\end{array}$ & $\begin{array}{l}6(20.7) \\
4(13.8) \\
15(51.7) \\
4(13.8)\end{array}$ & $\begin{array}{l}3(18.7) \\
2(12.5) \\
10(62.5) \\
1(6.3)\end{array}$ & 0.855 \\
\hline $\begin{array}{l}\text { Pathological type } \\
\text { Intestinal } \\
\text { Diffuse } \\
\text { Mixed }\end{array}$ & $\begin{array}{l}18(62.1) \\
9(31.0) \\
2(6.90)\end{array}$ & $\begin{array}{l}9(56.2) \\
5(31.3) \\
2(12.5)\end{array}$ & 0.810 \\
\hline $\begin{array}{l}\text { HER2 status } \\
\qquad \mathrm{IHC}(3+) \\
\mathrm{IHC}(2+) \text { and FISH (+) }\end{array}$ & $\begin{array}{l}25(86.2) \\
4(13.8)\end{array}$ & $\begin{array}{l}\text { I5 (93.7) } \\
\text { I (6.3) }\end{array}$ & $0.44 I$ \\
\hline
\end{tabular}

group, the ORR was $72.4 \%$ (21/29), and the DCR was $89.7 \%(26 / 29)$. In the control group, the ORR was $43.8 \%$ (7/16), and the DCR was $87.5 \%(14 / 16)$. All patients with CR had stage II disease.

\section{Tumor Resolution, Surgical Margin, and Tumor Downstaging}

Forty-five patients underwent radical gastrectomy with D2 dissection. Among the 29 patients in the experimental group, 28 underwent R0 resection, 1 underwent R1 resection, and
Table 2 Curative Effect Among 45 Patients with Operable HER2-Positive Advanced Gastric Cancer

\begin{tabular}{|l|l|l|l|}
\hline Group Efficacy & $\begin{array}{l}\text { T + FLOT, } \\
\text { n (\%) }\end{array}$ & $\begin{array}{l}\text { FLOT, } \\
\text { n (\%) }\end{array}$ & P value \\
\hline $\begin{array}{l}\text { Imaging evaluation } \\
\text { CR }\end{array}$ & $5(17.2)$ & $1(6.3)$ & 0.226 \\
PR & $16(55.2)$ & $6(37.5)$ & \\
SD & $5(17.2)$ & $7(43.7)$ & \\
\hline Pathological evaluation & & & \\
TRG Ia & $5(17.2)$ & $I(6.3)$ & 0.468 \\
TRGIb & $8(27.6)$ & $3(18.7)$ & \\
TRG2 & $9(31.0)$ & $5(31.3)$ & \\
TRG3 & $7(24.1)$ & $7(43.7)$ & \\
\hline
\end{tabular}

the $\mathrm{R} 0$ resection rate was $96.5 \%$. Among the 16 patients in the control group, 15 underwent R0 resection, 1 underwent $\mathrm{R} 1$ resection, and the R0 resection rate was $93.7 \%$. The results of pathological evaluation of patients in the two groups are shown in Table 2. The univariate analysis showed that TRG was correlated with pathological differentiation ( $\mathrm{P}=0.012)$, ECOG performance status $(\mathrm{P}=0.019)$, clinical stage $(P=0.002)$, pathological type $(P=0.001)$, and HER2 expression status $(\mathrm{P}=0.011)$. Patients with advanced gastric cancer with high pathological differentiation, ECOG performance score 0 , stage II, pathological intestinal type, and HER2 (3+) achieved better curative effect. See Table 3 that shows the descending phase of the tumor after neoadjuvant treatment. It was found that the $\mathrm{cN}+/ \mathrm{ypN} 0$ of the experimental group (T+FLOT) was $53.8 \%$, while that of the control group (FLOT) was only $35.7 \%$.

\section{Adverse Effects}

During treatment, no treatment-related deaths were recorded. The adverse effects observed in the two treatment groups were similar. The main grade III-IV adverse effects were hematotoxicity and digestive tract toxicity, which could be well controlled through prophylactic treatment. Further details are shown in Table 4. Three patients in the experimental group required dose adjustment for docetaxel and oxaliplatin. One patient was found to be allergic to oxaliplatin during the fourth cycle of treatment, which was relieved after treatment, while 2 patients in the control group required dose adjustment for docetaxel and oxaliplatin. In the experimental group, there was 1 case of abdominal infection and 1 case of delayed wound healing. In the control group, there were no cases of abdominal infection; however, there were 3 cases of delayed wound 
Table 3 Down Staging of 45 Patients with Resectable HER2+ Advanced Gastric Cancer

\begin{tabular}{|c|c|c|c|c|}
\hline Group & T+FLOT (n) & & FLOT (n) & \\
\hline Stage & Pre & Post & Pre & Post \\
\hline ॥ & $\begin{array}{l}\text { сT2N2MO } \\
\text { сT3NIMO } \\
\text { cT4aNOMO }\end{array}$ & $\begin{array}{l}\text { урTONOMO' } \\
\text { 'PTINOMO' } \\
\text { 'PTONOMO' } \\
\text { 'PTINOMO' } \\
\text { yPTONOMO² }\end{array}$ & $\begin{array}{l}\text { cT3NIMO² } \\
\text { cT4aNOMO' }\end{array}$ & $\begin{array}{l}\text { уPTINOMO' } \\
\text { yPT2NOMO' } \\
\text { 'РTONOMO' }\end{array}$ \\
\hline III & $\begin{array}{l}\text { сT3N2M0' } \\
\text { сT3N3aM05 } \\
\text { cT4aNIM0' } \\
\text { cT4aN2M0' } \\
\text { cT4aN3aM0 } \\
\text { cT4bN0M0' } \\
\text { cT4bN2M0 }\end{array}$ & 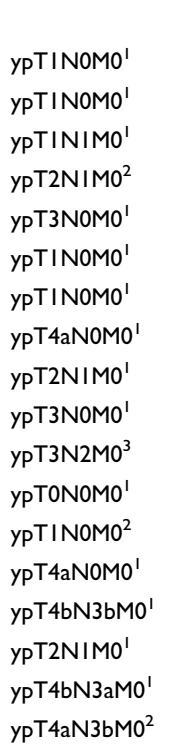 & $\begin{array}{l}\text { сT4aN3aM0 } \\
5 \\
\text { cT4bNOM0' } \\
\text { cT4bN2M0 } 2 \\
\text { cT4bN3aMo' }\end{array}$ & 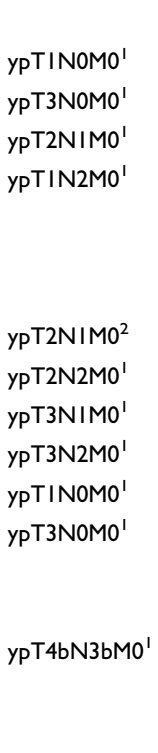 \\
\hline
\end{tabular}

healing. Postoperatively, 40 patients were treated with the original protocol, and 5 patients with PD were treated with the mFOLFOX6 protocol. Color Doppler echocardiography was performed every 3 months to evaluate left ventricular ejection fraction, which was found to be within the normal range for all patients.

\section{Survival Analysis}

As of September 1, 2019, 10 patients had died; 6 in the experimental group and 4 in the control group. The median follow-up time was 24.3 (range, 10-47) months and the 2-year OS was $77.1 \%$ (Figure 2). The two-year survival rates of the experimental and control groups were $78.1 \%$ and $73.9 \%$, respectively $(\mathrm{P}=0.932)$ (Figure 3 ). Single factor analysis showed that OS was related to pathological differentiation ( $\mathrm{P}=0.000)$, ECOG performance score $(\mathrm{P}=0.000)$, tumor location $(\mathrm{P}=0.000)$, clinical stage $(\mathrm{P}=0.008)$, pathological type $(\mathrm{P}=0.000)$, and TRG $(\mathrm{P}=0.000)$. Patients with better pathological differentiation, ECOG performance score 0 , tumor in the gastric antrum, stage II disease, intestinal type, and tumor downstaging had better prognoses.
Table 4 Adverse Effects of Neoadjuvant Therapy Among 45 Patients with Advanced Gastric Cancer

\begin{tabular}{|l|l|l|l|l|}
\hline \multirow{2}{*}{ Group Adverse Effects } & \multicolumn{2}{|l|}{ T + FLOT, n (\%) } & \multicolumn{2}{l|}{ FLOT, n (\%) } \\
\cline { 2 - 5 } & I-IV & III-IV & I-IV & III-IV \\
\hline Agranulocytosis & $26(89.6)$ & $6(20.7)$ & I4 (87.5) & $3(18.7)$ \\
Agranulocytic fever & $\mathrm{I}(3.4)$ & $\mathrm{I}(3.4)$ & $\mathrm{I}(6.3)$ & $\mathrm{I}(6.3)$ \\
Anemia & $10(34.3)$ & $0(0)$ & $6(37.5)$ & $\mathrm{I}(6.3)$ \\
Thrombocytopenia & $15(5 \mathrm{I} .7)$ & $2(6.9)$ & $7(43.7)$ & $0(0)$ \\
Peripheral neurotoxicity & $23(79.3)$ & $\mathrm{I}(3.4)$ & $\mathrm{I}(81.2)$ & $\mathrm{I}(6.3)$ \\
Nausea & $19(65.5)$ & $\mathrm{I}(3.4)$ & $\mathrm{II}(68.7)$ & $\mathrm{I}(6.3)$ \\
Vomiting & $15(51.7)$ & $\mathrm{I}(3.4)$ & $9(56.2)$ & $\mathrm{I}(6.3)$ \\
Lethargy & $\mathrm{II}(37.9)$ & $0(0)$ & $9(56.2)$ & $0(0)$ \\
Mucositis & $3(10.3)$ & $0(0)$ & $2(12.5)$ & $0(0)$ \\
Diarrhea & $2(6.9)$ & $0(0)$ & $0(0)$ & $0(0)$ \\
Anaphylaxis & $\mathrm{I}(3.4)$ & $\mathrm{I}(3.4)$ & $0(0)$ & $0(0)$ \\
\hline
\end{tabular}

\section{Discussion}

Gastric cancer is a global public health problem. Advanced gastric cancer is more common in China. The 5-year survival rate is only $30-50 \%$ after surgical treatment. Therefore, it is necessary to identify better treatment modalities to further improve the prognosis of patients. At present, neoadjuvant therapy can be the first choice for advanced gastric cancer, and previous studies have confirmed that it can improve the survival of patients. ${ }^{9}$ Trastuzumab is the first drug that has been shown to significantly improve the prognosis of patients with metastatic gastric cancer and has not increased the side effects of chemotherapy. ${ }^{10}$ In HER2+ breast cancer, the addition of $\mathrm{T}$ to standard neoadjuvant chemotherapy significantly improved both, pathological complete response rates and event-free survival. ${ }^{11}$ However, the question over whether the $\mathrm{T}$ in perioperative chemotherapy may further improve treatment outcomes in patients with localized or locally advanced resectable GC remains one of the major open questions in the current management of GC patients. Although a number of ongoing clinical studies have attempted to answer this question, the final results have not been reported. In addition, as not all patients are eligible for treatment with FLOT, there is limited information on FLOT outside of Germany at present. Hospitalization for toxicity occurred in $25 \%$ in Germany's clinical trial. ${ }^{3}$ Therefore, it is still unknown whether trastuzumab combined with FLOT is suitable for perioperative treatment of HER-2-positive advanced gastric cancer. This study confirmed that the ORR and DCR of the FLOT regimen in the treatment of HER2-positive advanced gastric cancer were $43.8 \%$ and $87.5 \%$, respectively, while the $\mathrm{T}+$ FLOT regimen in the experimental group could significantly improve the ORR of 


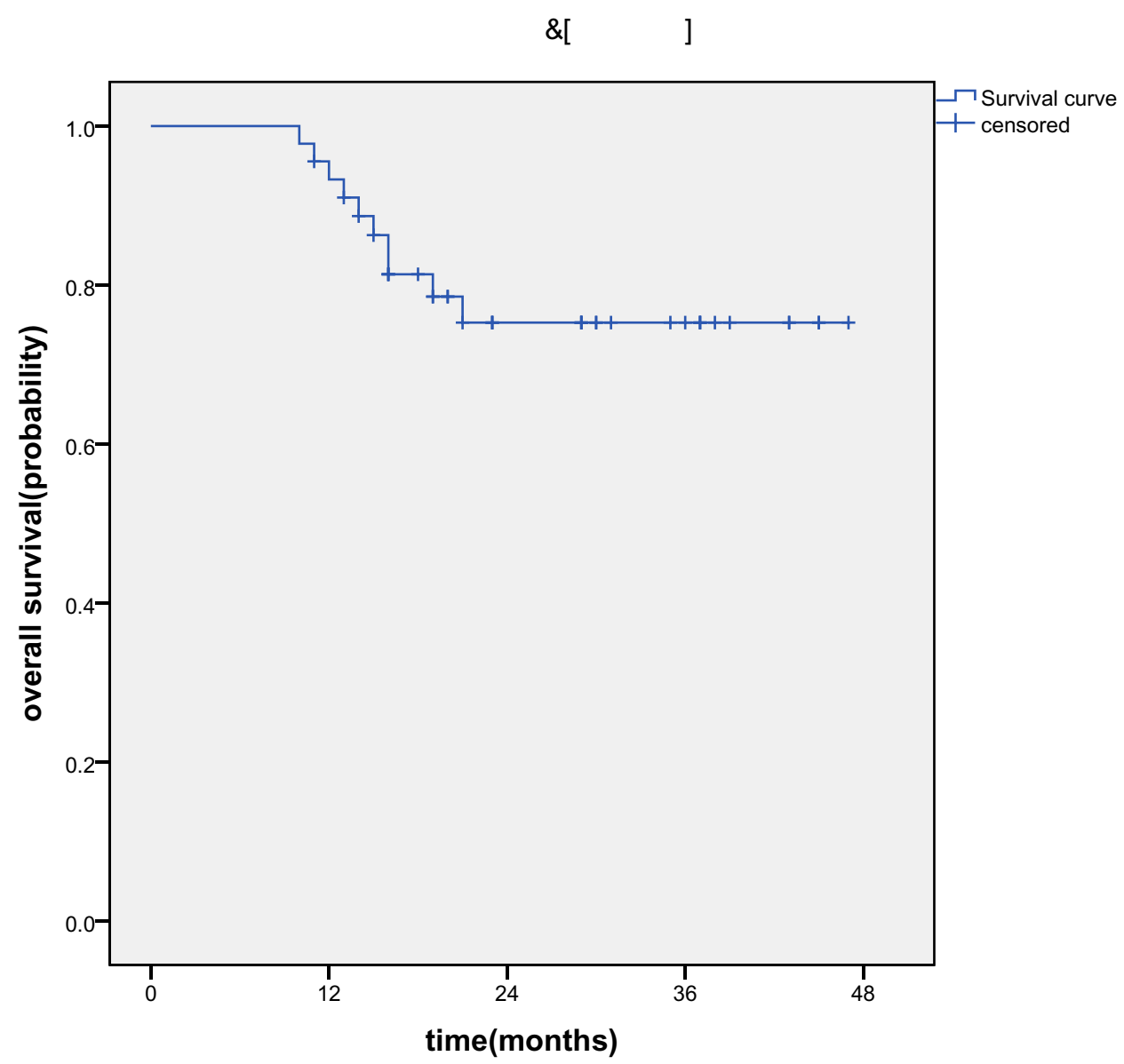

Figure 2 Overall survival time of 45 patients with operable HER2-positive advanced gastric cancer.

such patients, reaching $72.4 \%$, although the DCR was similar (only 89.7\%). However, the primary purpose of neoadjuvant treatment was tumor downstaging, which could yield more surgical opportunities among patients; thus, the T + FLOT regimen was more effective in improving the ORR than chemotherapy alone. Although there was no statistically significant difference, this may be related to the fewer number of cases in the control group. A study conducted in South Korea showed that the DCR of tegio combined with docetaxel (for 1 week) was $74.4 \%$ and the $\mathrm{R} 0$ resection rate was $97.7 \%{ }^{12}$ while the $\mathrm{R} 0$ resection rate of the $\mathrm{T}+$ FLOT regimen was $96.5 \%$, similar to the results of the previous study. A Spanish study showed that the ORR and DCR for trastuzumab combined with the XELOX regimen in the treatment of advanced gastric cancer patients were $46.7 \%$ and $77.8 \%$, respectively. ${ }^{13}$ The curative effect was worse than that of our study. In addition, we found that the $\mathrm{cN}+/ \mathrm{ypN} 0$ of the experimental group was $53.8 \%$, while the $\mathrm{cN}+/ \mathrm{ypN} 0$ of the control group was only $35.7 \%$. Previous research found that, in patients with gastric cancer who underwent preoperative therapy, we found similar OS in cN0/ypN0 and cN+/ypN0 patients. Because ypN + patients had poor OS, achieving ypN0 status is an important hallmark demonstrating the effectiveness of preoperative therapy for gastric cancer. ${ }^{14}$ When administering neoadjuvant therapy including trastuzumab for those with HER-2 positive breast cancer, the prognosis of the patients with $\mathrm{cN}+/ \mathrm{ypN} 0$ was better than that of the patients with $\mathrm{cN}+/ \mathrm{ypN}+{ }^{15}$ however, the value was still unclear in those with operable HER-2-positive advanced gastric cancer. The number of cases in this retrospective study is relatively small; however, based on preliminary data, we found that the $\mathrm{CN}+/ \mathrm{ypN} 0$ of $\mathrm{T}$ combined with FLOT is more advantageous. Thus, $\mathrm{T}+$ FLOT may be the best combination treatment modality for resectable HER2positive advanced gastric cancer, yielding more surgical opportunities among patients.

The degree of tumor regression is an important index for evaluating the therapeutic effect of neoadjuvant therapy. Although clinical studies with small samples have confirmed that neoadjuvant concurrent radiotherapy and chemotherapy 


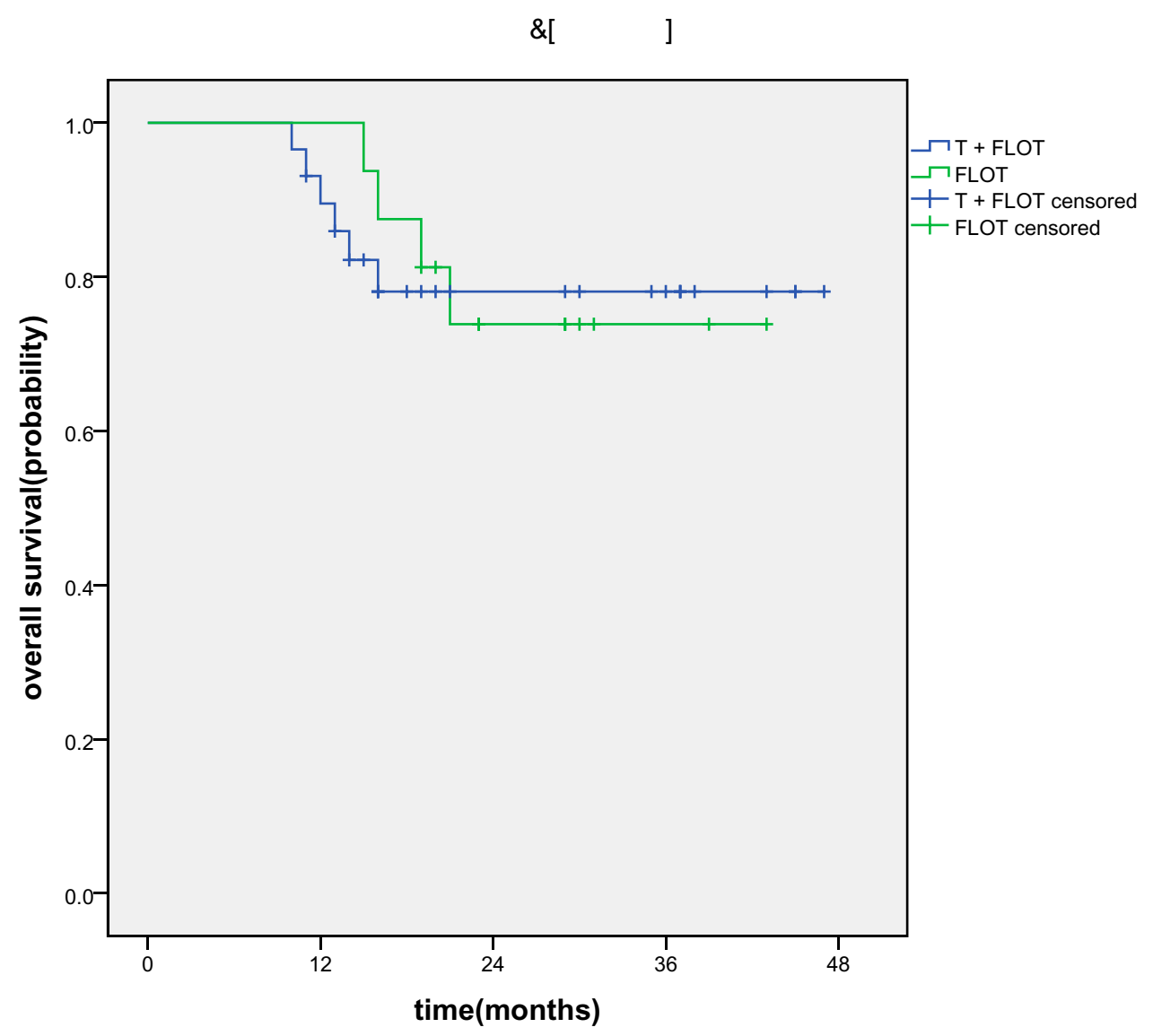

Figure 3 Overall survival time of 45 patients with operable HER2-positive advanced gastric cancer in different treatment groups.

can reduce the stage of locally advanced gastric cancer, improve the R0 resection rate and PCR rate. For example, phase II clinical studies have shown that neoadjuvant concurrent radiotherapy and chemotherapy (SOX scheme) can treat locally advanced gastric cancer; one study showed that in 24 patients $(67 \%)$ who received radical surgery, the PCR rate was $13.9 \%$, and the median survival time was 30.3 months. ${ }^{16}$ Preoperative chemoradiation may be an effective treatment strategy among patients with locally advanced gastric adenocarcinoma, However, it still needs to be confirmed by a large sample study. In addition, the value of neoadjuvant radiotherapy and chemotherapy in patients with HER-2-positive advanced gastric cancer is not clear, especially if the combination of trastuzumab treatment increases the cardiotoxicity, so this study only uses the neoadjuvant treatment mode based on trastuzumab. The phase II NeoFLOT clinical study conducted in Germany showed that both the TRG1a and TRG1b were $20 \%$ for the FLOT regimen as neoadjuvant chemotherapy for resectable gastric or gastroesophageal junction adenocarcinoma, and the proportion of patients with pathological intestinal type was higher. ${ }^{17}$ In our study, we found that the TRG1a and TRG1b in the control group were lower $(6.3 \%$ and $18.7 \%$, respectively), which may be related to the specific population of HER2-positive patients. Previous studies showed that the prognosis of patients with HER2-positive gastric cancer was worse. In addition, in the FLOT4-AIO study, the pCR rate of the FLOT regimen was significantly higher than that of the ECF/ECX protocol ( $16 \%$ and $6 \%$, respectively) $(\mathrm{P}=0.02) .{ }^{18}$ In this study, all the patients in the experimental group underwent radical gastrectomy combined with D2 dissection. Except for one case of gastric cancer with microscopically positive esophageal surgical margin, all the other patients received R0 resection; $17.2 \%$ of the patients achieved TRG1a and $44.8 \%$ achieved TRG1. Therefore, the $\mathrm{T}+$ FLOT regimen is not only superior to that determined in the previous clinical study but also superior to the control group in this study in the treatment of operable HER2positive advanced gastric cancer. We also found that patients with advanced gastric cancer with high pathological differentiation, ECOG performance score of 0, stage II disease, pathological intestinal type, and HER2 (3+) had better 
prognoses. So can other targeted drugs combined with chemotherapy improve the pathological remission of patients? In the ST03 clinical study, bevacizumab combined with ECX did not improve the effective rate and pathological tumor response rate of the patients ( $42 \%$ and $30 \%$, respectively), similar to ECX alone $(\mathrm{P}>0.05) .{ }^{19}$ However, this study confirmed that trastuzumab combined with the FLOT regimen can improve the degree of tumor regression of HER2positive gastric cancer in the resectable advanced stage and yield more clinical benefits among patients.

Regarding adverse effects, the incidence of hematotoxicity and gastrointestinal toxicity in the two groups was high, but the patients tolerated treatment well and successfully completed the perioperative treatment, as observed in previous studies, such as the FLOT4-AIO clinical study. The main grade 3 and 4 adverse effects were leukopenia (36\%) and nausea and vomiting $(3 \%) .{ }^{18}$ Adding trastuzumab on the basis of FLOT did not increase the adverse effects. Cardiac function was normal among the patients. Although there was no treatment-related deaths or increased risk of surgery in this study, all patients tolerated the medication and successfully completed the perioperative treatment. However, this study had a small sample and retrospective design. We are currently awaiting the results of the INNOVATION and PETRARCA clinical studies. The main purpose of perioperative treatment is to improve the survival of patients except for downstaging. The median follow-up time in this study was 2 years, and the two-year OS was $78.1 \%$. The 3-year survival rates in the previous FLOT4-AIO and Fiteni clinical studies were $57 \%$ and $60 \%$, respectively, which were superior to the $45 \%$ identified in the MAGIC clinical study. ${ }^{2,3,20}$ In this study, a specific population with HER2 positive was selected. The median follow-up time was about 2 years, and the two-year OS was 77.1\%. The two-year survival rates for the experimental and control groups were $78.1 \%$ and $73.9 \%$, respectively $(\mathrm{P}=0.932)$. Adding trastuzumab based on FLOT did not improve the prognosis of resectable HER2-positive advanced gastric cancer, which was related to the short follow-up time of this study, most of the patients in this study were stage III patients, a small sample retrospective study, and the case selection may be biased. Although the $\mathrm{T}+$ FLOT regimen yields a trend of survival improvement, there was no statistically significant difference, and thus requires longer follow-up. Univariate analysis showed that patients with better pathological differentiation, ECOG performance score of 0, antral tumor, stage II disease, intestinal type, and tumor regression had better prognoses, similar to the results of previous studies. As the survival data of this study were not long term, further follow-up is required to confirm that trastuzumab combined with the FLOT regimen can improve the prognosis of patients with resectable HER2-positive advanced gastric cancer; however, the preliminary study shows that the $\mathrm{T}+$ FLOT regimen has survival advantages and can yield survival benefits among such patients.

In a word, trastuzumab combined with FLOT can be used as a neoadjuvant treatment modality for resectable advanced HER2-positive gastric cancer. This regimen did not only improve the ORR of patients but also yield good tumor regression, improve the survival time of patients, and yield good safety, which are worthy of clinical promotion. Considering that the research is retrospective, and the number of cases is small, more clinical studies are still needed to confirm these findings. The international multicenter INNOVATION and PETRARCA studies are ongoing, in hopes of identifying more relevant clinical evidence in the future.

\section{Ethics and Consent Statement}

This study was approved by Ethics Committee of Peking University Shenzhen Hospital. And all the protocol used in the present study was approved by the Institutional Review Boards of all participating centers. Moreover, all these procedures were performed in accordance with the ethical standards established in the Declaration of Helsinki. All patients provided written informed consent before starting the study.

\section{Funding}

This work was supported by Natural Science Foundation of Guangdong Province (No. 2018A0303100026); Shenzhen Science and Technology Innovation Committee Fund (GJHZ20180420180754917) and Sanming Project of Shenzhen (SZSM201612041).

\section{Disclosure}

The authors report no conflicts of interest in this work.

\section{References}

1. Song Z, Wu Y, Yang J, et al. Progress in the treatment of advanced gastric cancer. Tumour Biol. 2017;39(7):1010428317714626. doi: $10.1177 / 1010428317714626$

2. Choi AH, Kim J, Chao J. Perioperative chemotherapy for resectable gastric cancer: MAGIC and beyond. World J Gastroenterol. 2015;21 (24):7343-7348. doi:10.3748/wjg.v21.i24.7343 
3. Al-Batran SE, Homann N, Pauligk C, et al. Perioperative chemotherapy with fluorouracil plus leucovorin, oxaliplatin, and docetaxel versus fluorouracil or capecitabine plus cisplatin and epirubicin for locally advanced, resectable gastric or gastro-oesophageal junction adenocarcinoma (FLOT4): a randomised, Phase 2/3 trial. Lancet. 2019;393(10184):1948-1957. doi:10.1016/S0140-6736(18)32557-1

4. Pereira MA, Ramos MFKP, Dias AR, et al. Expression profile of markers for targeted therapy in gastric cancer patients: HER-2, microsatellite instability and PD-L1. Mol Diagn Ther. 2019;23 (6):761-771. doi:10.1007/s40291-019-00424-y

5. Xu B, Huang C, Yang X, et al. Significance and prognostic role of human epidermal growth factor receptor 2 and RAB1A expression in gastric cancer. Oncol Lett. 2018;15(4):5185-5192. doi:10.3892/ol.2018.7992

6. Bang YJ, Van Cutsem E, Feyereislova A, et al. Trastuzumab in combination with chemotherapy versus chemotherapy alone for treatment of HER2-positive advanced gastric or gastro-oesophageal junction cancer (ToGA): a Phase 3, open-label, randomised controlled trial. Lancet 2010;376(9742):687-697. doi:10.1016/S0140-6736(10)61121-X

7. Tabernero J, Hoff PM, Shen L, et al. Pertuzumab plus trastuzumab and chemotherapy for HER2-positive metastatic gastric or gastro-oesophageal junction cancer (JACOB): final analysis of a double-blind, randomised, placebo-controlled phase 3 study. Lancet Oncol. 2018;19(10):1372-1384. doi:10.1016/S1470-2045(18)30481-9

8. Gong J, Liu T, Fan Q, et al. Optimal regimen of trastuzumab in combination with oxaliplatin/capecitabine in first-line treatment of HER2-positive advanced gastric cancer (CGOG1001): a multicenter, phase II trial. BMC Cancer. 2016;16:68. doi:10.1186/ s12885-016-2092-9

9. Wagner AD, Lordick F, Grabsch HI, et al. Multidisciplinary management of stage II-III gastric and gastro-oesophageal junction cancer. Eur J Cancer. 2020;124:67-76. doi:10.1016/j.ejca.2019.09.006

10. Bonelli P, Borrelli A, Tuccillo FM, et al. Precision medicine in gastric cancer. World J Gastrointest Oncol. 2019;11(10):804-829. doi:10.4251/ wjgo.v11.i10.804

11. Shao Z, Pang D, Yang H, et al. efficacy, safety, and tolerability of pertuzumab, trastuzumab, and docetaxel for patients with early or locally advanced ERBB2-positive breast cancer in Asia: the PEONY phase 3 randomized clinical trial. JAMA Oncol. 2019. doi:10.1001/ jamaoncol.2019.3692

12. Kim YW, Kim MJ, Ryu KW, et al. A phase II study of perioperative S-1 combined with weekly docetaxel in patients with locally advanced gastric carcinoma: clinical outcomes and clinicopathological and pharmacogenetic predictors for survival. Gastric Cancer. 2016;19(2):586-596. doi:10.1007/s10120-015-0490-3
13. Rivera F, Romero C, Jimenez-Fonseca P, et al. Phase II study to evaluate the efficacy of Trastuzumab in combination with Capecitabine and Oxaliplatin in first-line treatment of HER2-positive advanced gastric cancer: HERXO trial. Cancer Chemother Pharmacol. 2019;83(6):1175-1181. doi:10.1007/s00280019-03820-7

14. Coimbra FJF, de Jesus VHF, Ribeiro HSC, et al. Impact of ypT, ypN, and adjuvant therapy on survival in gastric cancer patients treated with perioperative chemotherapy and radical surgery. Ann Surg Oncol. 2019;26(11):3618-3626. doi:10.1245/s10434-019-07454-0

15. Qu LT, Peters S, Cobb AN, et al. Considerations for sentinel lymph node biopsy in breast cancer patients with biopsy proven axillary disease prior to neoadjuvant treatment. Am J Surg. 2018;215 (3):530-533. doi:10.1016/j.amjsurg.2017.11.015

16. Liu X, Li G, Long Z, et al. Phase II trial of preoperative chemoradiation plus perioperative SOX chemotherapy in patients with locally advanced gastric cancer. J Surg Oncol. 2018;117(4):692-698. doi:10.1002/jso. 24917

17. Schulz C, Kullmann F, Kunzmann V, et al. NeoFLOT: multicenter phase II study of perioperative chemotherapy in resectable adenocarcinoma of the gastroesophageal junction or gastric adenocarcinoma-Very good response predominantly in patients with intestinal type tumors. Int J Cancer. 2015;137(3):678-685. doi:10.1002/ijc. 29403

18. Al-Batran SE, Hofheinz RD, Pauligk C, et al. Histopathological regression after neoadjuvant docetaxel, oxaliplatin, fluorouracil, and leucovorin versus epirubicin, cisplatin, and fluorouracil or capecitabine in patients with resectable gastric or gastro-oesophageal junction adenocarcinoma (FLOT4-AIO): results from the phase 2 part of a multicentre, open-label, randomised phase 2/3 trial. Lancet Oncol. 2016;17(12):1697-1708. doi:10.1016/S1470-2045(16)30531-9

19. Cunningham D, Stenning SP, Smyth EC, et al. Peri-operative chemotherapy with or without bevacizumab in operable oesophagogastric adenocarcinoma (UK Medical Research Council ST03): primary analysis results of a multicentre, open-label, randomised phase 2-3 trial. Lancet Oncol. 2017;18(3):357-370. doi:10.1016/S14702045(17)30043-8

20. Cunningham D, Allum WH, Stenning SP, et al. Perioperative chemotherapy versus surgery alone for resectable gastroesophageal cancer. $N$ Engl J Med. 2006;355(1):11-20. doi:10.1056/NEJMoa 055531

\section{Publish your work in this journal}

Cancer Management and Research is an international, peer-reviewed open access journal focusing on cancer research and the optimal use of preventative and integrated treatment interventions to achieve improved outcomes, enhanced survival and quality of life for the cancer patient.
The manuscript management system is completely online and includes a very quick and fair peer-review system, which is all easy to use Visit http://www.dovepress.com/testimonials.php to read real quotes from published authors. 